\title{
Encoding partially ordered information
}

\author{
RICHARD A. GRIGGS, DONALD M. KEEN, and SUSAN A. WARNER \\ University of Florida, Gainesville, Florida 32611
}

\begin{abstract}
The typical distance effect for comparative judgments about ordered information (faster responses for nonadjacent comparisons than for adjacent comparisons) has not been found in recent experiments on processing highly complex 12-term partial orders. However, the rather complicated training and testing procedures used in these studies may have contributed to this failure to observe typical distance effects. The present experiment investigated the processing of a seven-term partial order in simpler experimental conditions similar to those employed in complete order studies. Results indicated that there were pronounced individual differences in encoding such information. Since only three subjects were judged to be using a valid representation of the partial order, no conclusions about the lack of distance effects in comparing partially ordered information could be drawn. Most subjects used memory representations that removed some or all of the indeterminacy in the partial order. It was concluded that most people do not have a F-econceived scheme for encoding partially ordered information.
\end{abstract}

In recent experiments using artificial (experimenterdefined) linear orders to study inferential reasoning abilities, subjects compared nonadjacent relations faster than adjacent relations (e.g., Pliske \& Smith, 1979; Potts, 1974; Woocher, Glass, \& Holyoak, 1978). Such a "distance effect" has occurred invariably for comparative judgments about linearly ordered information (see Potts, Banks, Kosslyn, Moyer, Riley, \& Smith, 1978, for a review of such effects). However, two studies on the processing of 12-term partial orders have found either no distance effect (Moeser \& Tarrant, 1977) or a reverse distance effect-faster responses for adjacents (Hayes-Roth \& Hayes-Roth, 1975).

Unlike complete linear orders, partial orders cannot be validly arranged in a linear array. Such orders must be represented in a physically multidirectional pattern. A sample partial order structure from the study by HayesRoth and Hayes-Roth (1975) is given in Figure 1. In this example, directed arcs representing the greaterthan relation connect the points that represent each letter. The transitivity property of the greater-than relation results in two types of true relationships in the structure, those between adjacent points connected by a single directed arc (e.g., $\mathrm{B}>\mathrm{J}$ ) and those between nonadjacent points connected indirectly by two or more directed arcs (e.g., B $>$ H). Two other types of relationships are present within the structure, false relations that are simply the converses of true relations (e.g., $\mathrm{J}>\mathrm{B}$ ) and indeterminate relations (e.g., $\mathrm{B}>\mathrm{K}$ ). It is the presence of the latter indeterminate relations that differentiate partial and complete orders.

This research was presented at the annual meeting of the Midwestern Psychological Association, May 1978, in Chicago, Illinois. The second author is now at Bell Laboratories, Indianapolis, Indiana. Requests for reprints should be sent to Richard Griggs, Department of Psychology, University of Florida, Gainesville, Florida 32611.

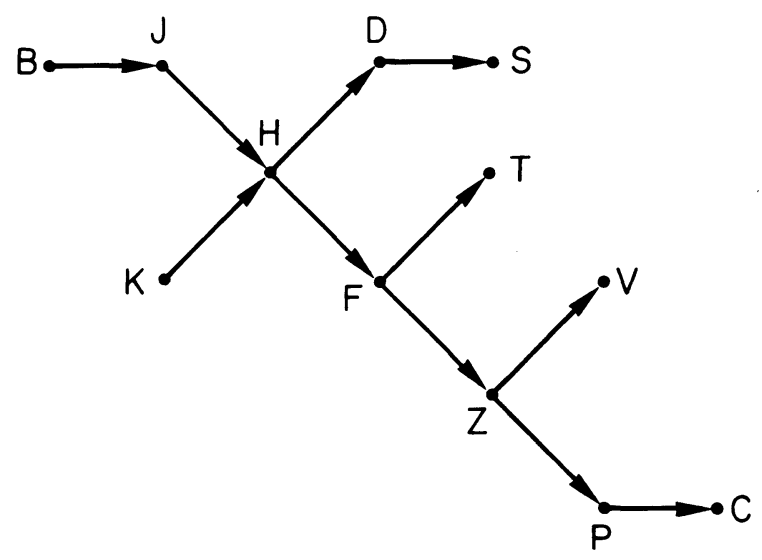

Figure 1. The 12-term partial order structure used by HayesRoth and Hayes-Roth (1975). (Reproduced with the authors' permission.)

Although highly probable, it is not clear that this indeterminacy in partial order structures is responsible for the failure to observe distance effects in comparative judgment studies using such information. The experimental procedures employed in complete and partial order studies have been very different. The partial order studies have used very elaborate learning and training phases on the adjacent relations before any overall testing. In contrast, the procedures that have been employed in complete order studies are very simple. Subjects usually study either the adjacent relations in some prose frame or the actual complete order for a self-paced period of time. This study period is followed by a true-false or two-alternative forced-choice test on all the possible relations in the order.

The present experiment investigated the processing of a smaller partial order under experimental conditions like those used in complete order studies. First, the partial order was presented in paragraph form, similar to that used in the initial complete order studies of 
Potts $(1972,1974)$. This mode of presentation should have also prevented the storage of the partially ordered information in multiple episodic traces (see Moeser \& Tarrant, 1977). In addition, a self-paced study/true-false test procedure like that used in most complete order studies was employed. The absence of a typical distance effect under these simplified conditions would indicate that the indeterminacy inherent in artificial partial orders necessitates processing strategies that do not result in the typical distance effect for comparative judgments.

\section{METHOD}

\section{Subjects}

Sixteen University of Florida undergraduates served as subjects in partial fulfillment of an introductory psychology course requirement.

\section{Materials}

The following paragraph describing a seven-term partial order was used.

"At the end of the term, a final exam was given in psychology class. When the instructor first looked at the exams, he noticed that Susan scored higher than Danny, Danny scored higher than Karen, and Karen scored higher than Linda. When the rest of the exams were graded, he saw that Larry scored higher than Karen, Karen scored higher than David, and David scored higher than Ellen. The instructor posted the seven final exam scores on his office door."

A 42-item true-false test on this paragraph was constructed. All test items were of the form "A scored higher than B," where A and B could be any of the seven students' names. Seventeen pairwise comparisons of the seven names are either stated in or deducible from the paragraph. These comparisons served as "true" items. Seventeen "false" test statements were constructed by reversing the order of the terms in the "true" items. Additionally, the eight comparisons whose truth value cannot be determined from the paragraph, such as "Susan scored higher than Larry," were included as "false" test items.

A questionnaire was given to each subject at the conclusion of the last test session. The subjects were asked to draw a representation of the ordering information described in the paragraph and to describe any strategies used in studying the paragraph and/or answering the test questions. They were also asked if they thought that the name "Karen" (mentioned twice in the paragraph) referred to the same student and if they realized that the two orders could be integrated into one larger structure.

\section{Apparatus}

All instructions, paragraphs, and questions were presented on a Perkins-Elmer 1100 video terminal. Two labeled keys on the terminal keyboard were used for responses. Stimulus presentation and response collection were controlled by a PDP-11/34 computer.

\section{Procedure}

Subjects were run individually. Some general study instructions preceded the presentation of the partial order paragraph. After a self-paced period of study, the subject was provided detailed test instructions. These instructions explained the nature of determinable "true" and "false" comparisons for this test. Test items were to be considered true if they were consistent with the information given in the paragraph and false if not consistent with this information. In addition, subjects were told that any statement whose truth value could not be determined from the information in the paragraph should be con- sidered false. These instructions made all indeterminate comparisons false.

Subjects were further instructed to indicate their truthvalue judgments by pressing the appropriate key. For one-half of the subjects, the left-hand key was labeled "True" and the right-hand key "False," while for the remaining subjects the labels were reversed. Subjects were also instructed to respond as quickly but as accurately as possible. The test instructions were followed by a self-paced randomized presentation of the test items. Three additional trials of self-paced study and truefalse testing followed the first test. The test instructions were repeated before each test. The subject was asked to complete the questionnaire following the last test.

\section{RESULTS}

\section{Proportion Correct Analyses}

The proportions correct for determinate and indeterminate comparisons were calculated for each trial. These means are given in Table 1. Performance on determinate comparisons increased from Trial 1 to Trial 2 but was uniformly high after the second trial. Performance on indeterminate comparisons, however, was uniformly low on all trials. Based on the high error rate for indeterminate comparisons, it is possible that many subjects did not have a valid functional representation of the information and therefore were not answering the indeterminate comparisons correctly. To check this hypothesis, the posttest questionnaire responses were examined.

On the posttest questionnaire, subjects were asked to draw their representation of the ordering information. Even though all 16 subjects indicated on the questionnaire that they realized that it was the same Karen mentioned twice in the paragraph and that the two orders could be integrated, only 3 of the 16 subjects drew a validly integrated seven-term structure (the two four-term orders crossing at the Karen node). A sample structure is given in Figure 2. Of the other 13 subjects, 5 represented the information as a complete linear order and 8 as either a semicollapsed partial order (i.e., incorrectly treating some but not all of the indeterminate comparisons as determinate comparisons) or two separate four-term orders.

When the drawing of each subject was verified against his last-trial responses, it was observed that the three subjects who drew a valid integrated structure answered the eight indeterminate test items correctly. The other 13 subjects did not answer all of these indeterminate items correctly, but rather answered in agreement with

Table 1

Mean Proportions Correct for Determinate (D) and Indeterminate (I) Comparisons for Each Trial

\begin{tabular}{|c|c|c|c|c|c|c|c|}
\hline \multicolumn{2}{|c|}{ Trial 1} & \multicolumn{2}{|c|}{ Trial 2} & \multicolumn{2}{|c|}{ Trial 3} & \multicolumn{2}{|c|}{ Trial 4} \\
\hline Mean & SE & Mean & $\mathrm{SE}$ & Mean & SE & Mean & SE \\
\hline .893 & .013 & .974 & .007 & .985 & .005 & .994 & .003 \\
\hline .625 & .043 & .625 & .043 & .711 & .040 & .679 & .041 \\
\hline
\end{tabular}




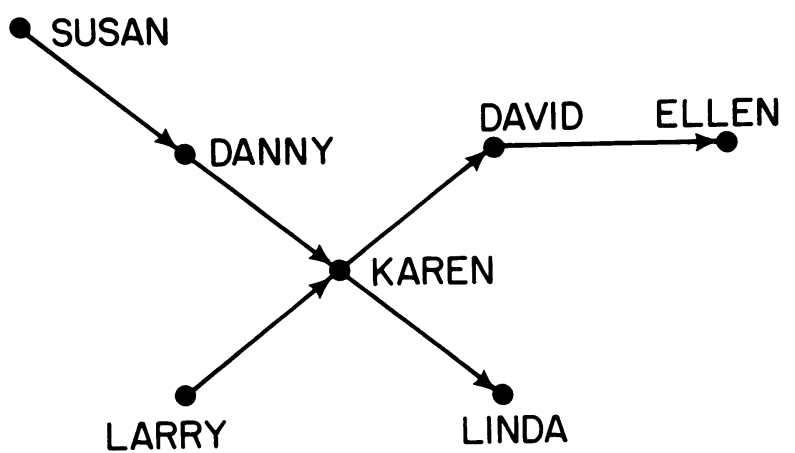

Figure 2. An example of a validly integrated representation of the partially ordered information used in the present experiment.

their variation of the seven-term structure. The two subjects who drew separate four-term orders responded as if they had used a semicollapsed partial order. Thus, based on performance on indeterminate comparisons and the drawings, there appeared to be three types of functional representations: invalid complete orders, invalid semicollapsed partial orders, and valid partial orders.

\section{Reaction Time Analyses}

Because of the individual differences in representing the partially ordered information, the subjects were divided into three groups for the reaction time analyses. The grouping was based on the inferred functional representation for each subject. Five subjects were in the complete order group, eight subjects were in the semicollapsed order group, and three subjects were in the valid partial order group.

To eliminate errant reaction times, all latencies greater than 2 standard deviations above the overall mean reaction time were truncated to that value. This procedure affected less than $2 \%$ of the data. Since reaction time consistently decreased over trials [mean(determinates) $=$ $3.76 \mathrm{sec}, 2.97 \mathrm{sec}, 2.59 \mathrm{sec}$, and $2.36 \mathrm{sec}$ for Trials $1-4$, respectively; mean(indeterminates) $=4.26 \mathrm{sec}, 3.10 \mathrm{sec}$, $2.87 \mathrm{sec}$, and $2.42 \mathrm{sec}$ for Trials $1-4$, respectively], Trial 4 reaction times formed the most stable data base. Because of this and the fact that the subject grouping was based on the Trial 4 accuracy data, all reaction time analyses were performed only on the Trial 4 data. Times for all responses were included in these analyses, since the overall error rate for this trial was less than $2 \%$ when the data were rescored in terms of the subjects' functional representations.

Analyses for subjects using complete orders. For the five subjects who used a complete order of the terms, each test item was classified as an adjacent or a nonadjacent in accordance with each subject's particular order. The mean reaction times for adjacent and nonadjacent comparisons were then calculated. As in the typical distance effect, responses for nonadjacents were faster than those for adjacents (mean $=2.09 \mathrm{sec}$ and mean $=2.58 \mathrm{sec}$, respectively). The data for four of the five subjects showed this difference. To further investigate this trend, the comparisons were divided according to a finer measure of distance, step size. Step size of a test item is defined in this study as the number of terms in the complete order that are between the two terms in the test item. Thus, an adjacent test item has a step size of 0 , and nonadjacent test items have step sizes greater than 0 . As step size increased, there was a general decrease in reaction time (mean $=2.58 \mathrm{sec}, 2.19 \mathrm{sec}$, $2.57 \mathrm{sec}, 1.75 \mathrm{sec}, 1.59 \mathrm{sec}$, and $1.64 \mathrm{sec}$, for Step Sizes $0-5$, respectively). A multiple-regression analysis revealed that only a linear trend was significant $[F(1,207)=10.02, p<.01]$. Thus, the results for these five subjects were very similar to those typically obtained in complete order studies.

Analyses for subjects using semicollapsed partial orders. Eight subjects used semicollapsed partial orders. Since different semicollapsed structures were used, the particular comparisons that were treated as indeterminate varied between subjects. Thus, the comparisons were classified in accordance with each subject's particular inferred structure. Three types of comparisons were used: adjacent, nonadjacent, and indeterminate. Indeterminates appeared to be the most difficult type of comparison (mean $=3.42 \mathrm{sec}$ ). The means for determinate comparisons were in the direction of a distance effect [mean(nonadjacents) $=2.30 \mathrm{sec}$ and mean(adjacents $)=2.49 \mathrm{sec}]$. This difference, however, was not significant $(p=.34)$. Five out of the eight subjects answered indeterminates the slowest, and six out of eight answered nonadjacents faster than adjacents.

Analyses for subjects using valid partial orders. For the three subjects who appeared to be utilizing a correctly integrated structure, the comparisons were divided into four types. In addition to adjacents and indeterminates, the nonadjacent determinates were divided into two types, simple nonadjacents (nonadjacent comparisons that involve terms from only one of the two presented four-term orders) and complex nonadjacents (nonadjacent comparisons that involve terms from both four-term orders). For example, "Susan scored higher than Karen" would be a simple nonadjacent, and "Susan scored higher than David" would be a complex nonadjacent. This division of nonadjacents is like that employed by Moeser and Tarrant (1977, Experiment 2). No significance tests were performed on these data, since the group size was so small.

Overall, simple nonadjacents were answered faster than adjacents (mean $=2.22 \mathrm{sec}$ and mean $=2.38 \mathrm{sec}$, respectively). The mean reaction time for complex nonadjacents (mean $=2.37 \mathrm{sec}$ ) was the same as the mean for adjacents. Indeterminates were again answered the slowest of all (mean $=2.77 \mathrm{sec})$. All three subjects were the slowest on indeterminates, but only one subject reflected the overall pattern for determinate compari- 
son types. Clearly, a typical distance effect was not present for these three subjects.

\section{DISCUSSION}

Based on the accuracy data for indeterminate comparisons and the posttest questionnaire drawings, definite individual differences in encoding the partially ordered information were observed. Three types of functional representations were used. Five subjects represented the information as an invalid complete order, eight subjects used a semicollapsed partial order, and only three subjects employed a correctly integrated structure. For the last two types of representation, indeterminate comparisons were the most difficult type of comparison to make. In addition, the closer the functional representation was to a complete order, the more similar the results were to those for complete orders. These findings seem to indicate that the indeterminacy of the partial order structure is responsible for the lack of distance effects when processing such information. However, since only three subjects appeared to use a valid representation of the information, no firm conclusions concerning the existence of distance effects for comparative judgments about partially ordered information can be made.

Most subjects did not appear to have a preconceived schema for processing partially ordered information. To varying degrees, these subjects attempted to fit such information into a complete order schema. Moeser (1979) has observed a similar tendency in her recent study of the acquisition of partially and completely ordered information. De Soto and his colleagues also observed this predilection for linear orders in their studies on the processing of social order structures (De Soto \& Albrecht, 1968).

It appears that in partial order experiments, subjects are asked to construct and use a representation for ordered information that is not normal for them. Ages, exam scores, and so on, can usually be positioned on one continuous dimension, and people have strong tendencies to represent order information in linear order structures even if it is not valid to do so. In a partial order experiment, however, the subject has to make this unidimensional representation multidirectional. Thus, because of this conflict with the normal way of processing order information, most subjects probably find the encoding of partially ordered information difficult. As Moeser and Tarrant (1977) observe, "patterns that appear to be very easy from the point of view of the experimenter may be very difficult to create from the point of view of the subject" (p. 656).

\section{REFERENCES}

De Soto, C. B., \& Albrecht, F. Cognition and social orderings. In R. P. Abelson, E. Aronson, W. J. McGuire, T. M. Newcomb, M. J. Rosenberg, \& P. H. Tannenbaum (Eds.), Theories in cognitive consistency: $A$ sourcebook. Chicago: Rand McNally, 1968.

HAyes-Roth, B., \& HAyes-Roth, F. Plasticity in memorial networks. Journal of Verbal Learning and Verbal Behavior, 1975, 14, 506-522.

Moese R, S. D. Acquiring complex partial orderings in comparison with acquiring similar-sized linear orderings. Memory \& Cognition, 1979, 7, 435-444.

Moeser, S. D., \& TARrant, B. L. Learning a network of comparisons. Journal of Experimental Psychology: Human Learning and Memory, 1977, 3, 643-659.

Pliske, R. M., \& Smith, K. H. Semantic categorization in a linear order problem. Memory \& Cognition, 1979, 7, 297-302.

PotTs, G. R. Information processing strategies used in the encoding of linear orderings. Journal of Verbal Learning and Verbal Behavior, 1972, 11, 727-740.

PotTs, G. R. Storing and retrieving information about ordered relationships. Journal of Experimental Psychology, 1974, 103, 431-439.

Potts, G. R., Banks, W. P., Kosslyn, S. M., Moyer, R. S., RILEY, C. A., \& Smith, K. H. Distance effects in comparative judgments. In N. J. Castellan \& F. Restle (Eds.), Cognitive theory (Vol. 3). Hillsdale, N.J: Erlbaum, 1978.

Woocher, F. D., Glass, A. L., \& Holyoak, K. J. Positional discriminability in linear orders. Memory \& Cognition, 1978, 6, 165-173.

(Received for publication March 8, 1980.) 